\title{
L'inventaire de la ligne touristique du Train Thur Doller Alsace de Cernay à Sentheim
}

An Inventory of the tourist line of the Thur Doller Alsace train from Cernay to Sentheim

Didier Leroy

\section{OpenEdition}

\section{Journals}

Édition électronique

URL : https://journals.openedition.org/rhcf/741

DOI : 10.4000/rhcf.741

Éditeur

Rails \& histoire

Édition imprimée

Date de publication : 1 novembre 2009

Pagination : 143-153

ISSN : 0996-9403

\section{Référence électronique}

Didier Leroy, "L'inventaire de la ligne touristique du Train Thur Doller Alsace de Cernay à Sentheim », Revue d'histoire des chemins de fer [En ligne], 40 | 2009, mis en ligne le 01 novembre 2011, consulté le 22 avril 2022. URL : http://journals.openedition.org/rhcf/741 ; DOI : https://doi.org/10.4000/rhcf.741 
Didier LEROY

\section{L'inventaire de la ligne touristique du Train Thur Doller Alsace de Cernay à Sentheim}

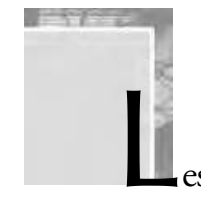

es communications d'Elisabeth Paillard, chercheur au Service régional de l'inventaire à la région Alsace, et de Romain Tricot, vice-président du Train Thur Doller Alsace, ont permis de dresser successivement l'inventaire des bâtiments et du matériel roulant de la ligne de la Doller. Pour ma part, j'aborderai un sujet peu connu qui peut se résumer dans l'expression «Inventaire des objets mobiliers et de petit matériel ", dénomination communément usitée par les anciennes compagnies et par la SNCF jusqu'au milieu des années 1970 pour désigner, dans les inventaires en place dans les gares, un certain nombre d'objets utilisés pour l'exploitation du chemin de fer. En effet, nous ne pouvons que constater la pauvreté des recherches et la quasi-inexistence d'inventaires dans ces domaines.

L'exemple de la ligne touristique de la vallée de la Doller, fermée au trafic voyageurs en 1967 et au trafic marchandises en 1972, est intéressant de ces points de vue, puisque nous y trouvons des types de gares de facture Compagnie de l'Est, Elsass-Lothringen (EL, 1871-1918) et Alsace-Lorraine (AL, 1918-1937) qui furent exploitées par ces trois compagnies ou réseaux puis par la SNCF. Les vicissitudes de l'histoire ont laissé leur empreinte sur les objets et sur leur conservation. La réactivation en 1976, sous la forme d'un 
chemin de fer touristique, de la section de Cernay à Sentheim $(13,65 \mathrm{~km})$ permet aujourd'hui de rappeler la mémoire de ces évolutions. Les figures jointes illustrent la dynamique patrimoniale qui est au cœur de notre association (fig. 1).

Figure 1 เ

Gare de Sentheim, le bâtiment-voyageurs.

${ }^{\odot}$ et cl. TTDA.

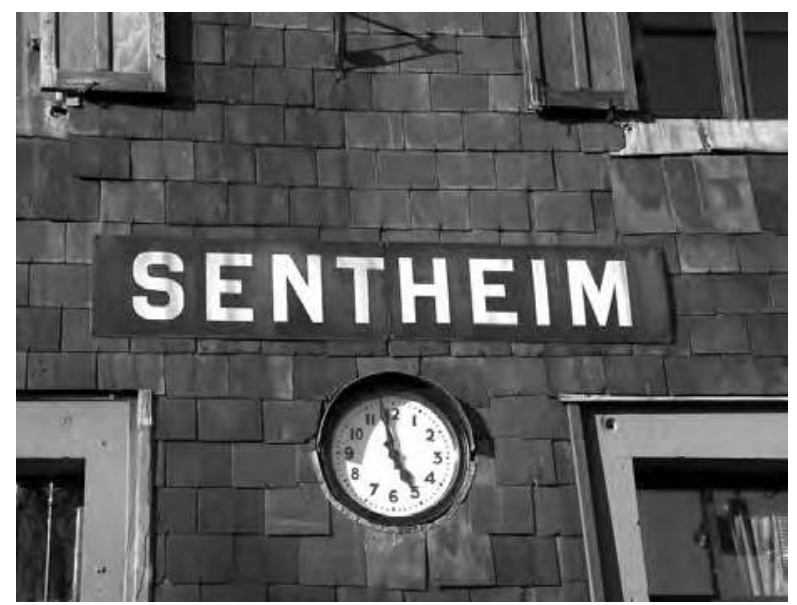

Mon propos est donc d'attirer l'attention sur les richesses patrimoniales qu'offrent les bâtiments restés, tant extérieurement qu'intérieurement, dans leur configuration d'origine, avec leur salle des pas perdus, leur guichet en bois vitré, leur salle d'attente, leurs locaux destinés à divers services. Dans ces intérieurs typiques de petites gares rurales, nous retrouvons la pendule mère de parquet, le régulateur donnant l'heure sur le quai, les lanternes éclairées au pétrole, le poêle à charbon, les plaques émaillées, le célèbre composteur en fonte, les tampons en cuivre aux encres indélébiles violette ou noire oléique et l'armoire pour les billets en carton aux dimensions standardisées $(57 \mathrm{~mm} \times 30 \mathrm{~mm}$ ) avec destination et taxation imprimées auxquels leur créateur, Thomas Edmonson (1752-1851), chef de gare à Milton, en Angleterre, a donné son nom en 1836 (fig. 2 à 4 ). 


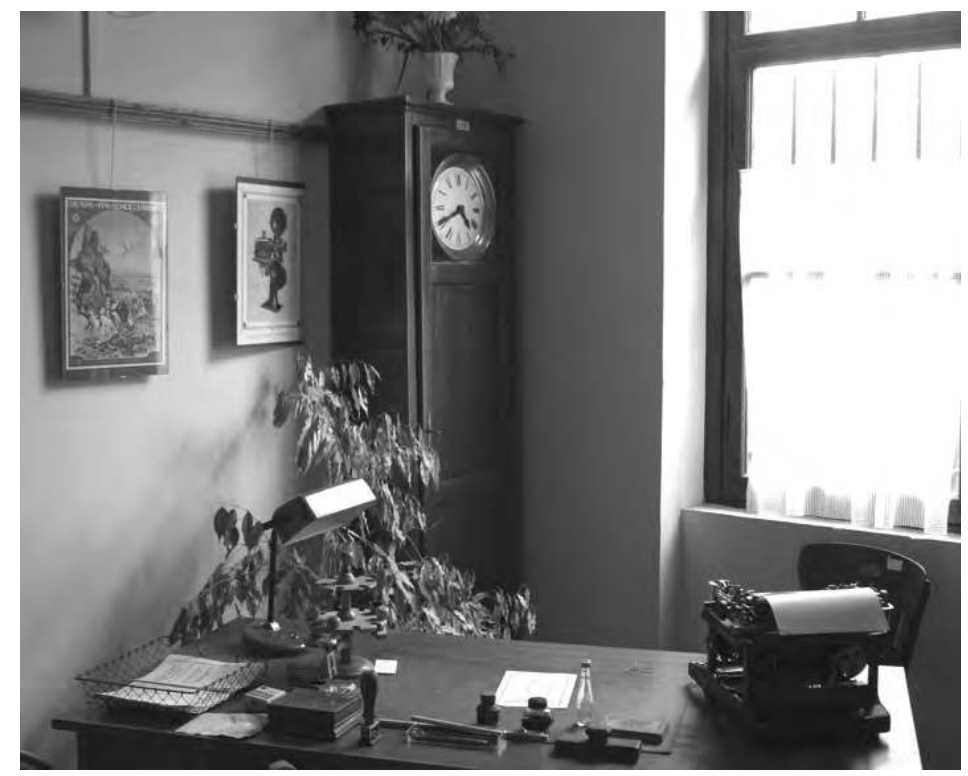

- Figure 2. Le bureau du chef de gare et sa pendule. ${ }^{\odot}$ et cl. TTDA.

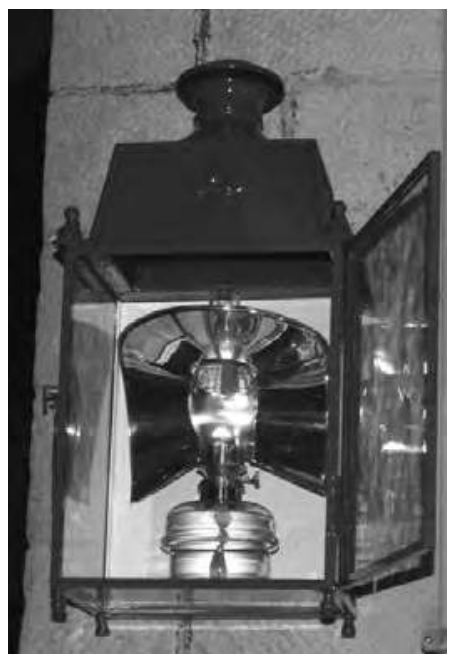

- Figure 3. Une lanterne type $A E^{\text {bis }}$. ๑ et cl. Didier Leroy.

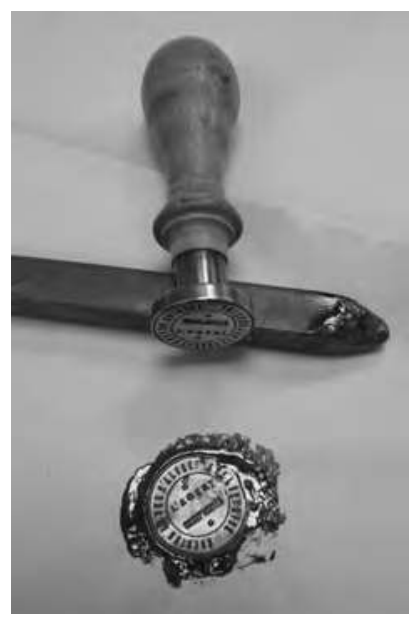

- Figure 4. Un cachet en cuivre $\mathrm{AL}$ et son empreinte dans la cire. $\odot$ et cl. Didier Leroy. 
Les passages à niveau avec leurs barrières oscillantes actionnées par un treuil, les cloches d'annonce Siemens, les bordures de quai en longrines métalliques, les barrières pivotantes rouges et blanches en treillis métallique fermant les cours de marchandises et les leviers d'aiguillages avec leur contrepoids estampillé AL font également partie intégrante d'un patrimoine que nous désirons non seulement conserver mais développer. L'association a ainsi restauré et remis en place un signal mécanique de type AL relativement rare, avec son avertissement s'effaçant horizontalement (fig. 5).

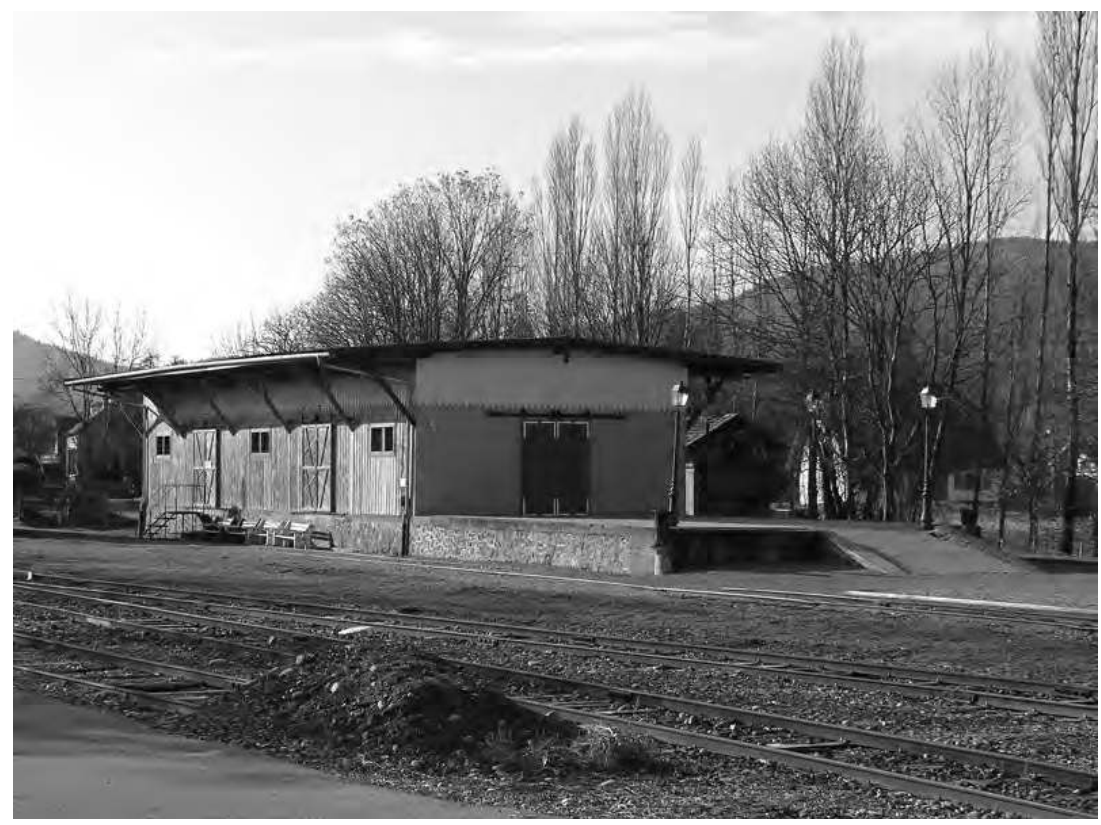

- Figure 5. Gare de Sentheim, halle à marchandises. ${ }^{\circledR}$ et cl. TTDA.

C'est par conséquent un ensemble cohérent - ce qui en fait sa richesse que propose le TTDA. Grâce à son parc voyageurs et marchandises, elle peut présenter au public un environnement ferroviaire des années 1920 avec un train mixte, un train omnibus voyageurs du milieu des années 1950 avec son fourgon et ses voitures modernisées du réseau Sud-Est à trois essieux (surnommées " trois-pattes »), ou encore un autorail rustique U 150 (le X 5852) (fig. 6). 


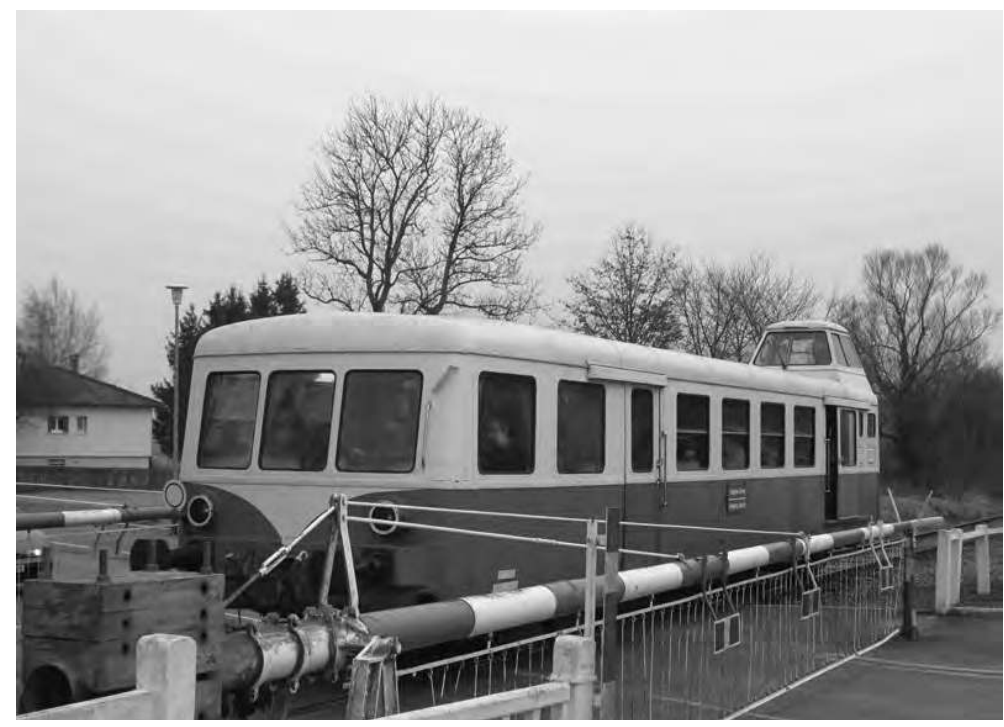

- Figure 6. Autorail $150 \mathrm{cv}$ à livrée rouge et crème, passage à niveau et barrières oscillantes. ${ }^{\odot}$ et cl. TTDA.

Trop souvent oubliés dans la muséographie ferroviaire, les hommes et femmes du rail sont évoqués également à travers leurs uniformes dont les casquettes, brodées de feuilles en canetille d'or et (ou) d'argent ou étoilées en fonction du grade de l'agent, sont emblématiques de leur temps.

En résumé, un bâtiment-voyageurs maintenu ou restauré extérieurement dans son état d'origine, mais dont l'intérieur, souvent limité à un grand espace vide doté d'un faux plafond, ne reflète plus ce qu'il était autrefois, n'incite guère à entrer dans une démarche didactique mettant en valeur un patrimoine vivant (fig. 7). Je souhaite donc sensibiliser les acteurs du patrimoine à l'urgence qu'il y a à trouver un cadre pour la protection d'une collection variée et cohérente, en insistant sur le petit matériel et le mobilier qui mériterait d'être protégé au titre des monuments historiques avant que les aléas du temps ne les fassent s'éparpiller, voire disparaître. Dans ce domaine, leur appui est indispensable tant en ce qui concerne la ligne de la Doller que pour des collections similaires, représentatives des petites et grandes compagnies ferroviaires. Cette aide, à la différence de la protection au titre des monuments historiques des matériels ferroviaires comme les locomotives, qui entraîne lors des travaux de restauration l'octroi par l'État d'importantes subventions, n'a pas de caractère 
financier puisque la restauration des pièces est due au talent de membres bénévoles de nos associations (fig. 8 et 9). C'est en termes de moyens de protection que se pose le problème.

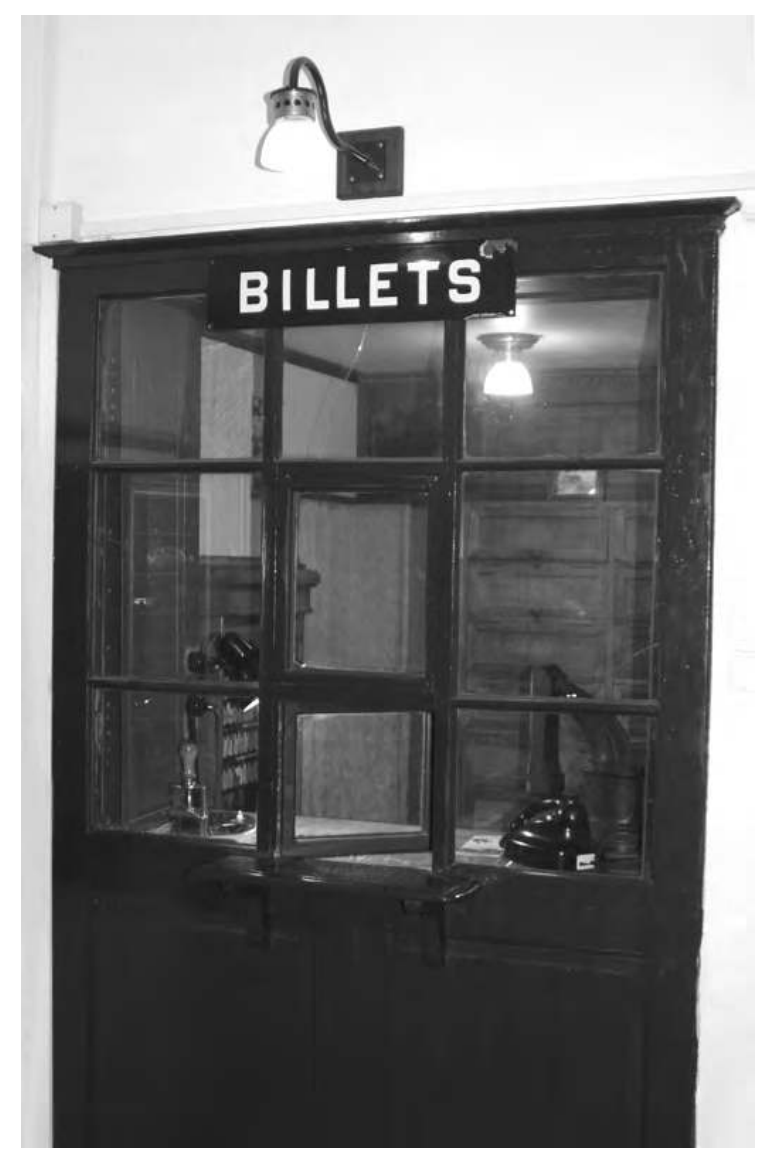

- Figure 7. Gare de Sentheim, intérieur, guichet. ๑ et cl. Didier Leroy. 


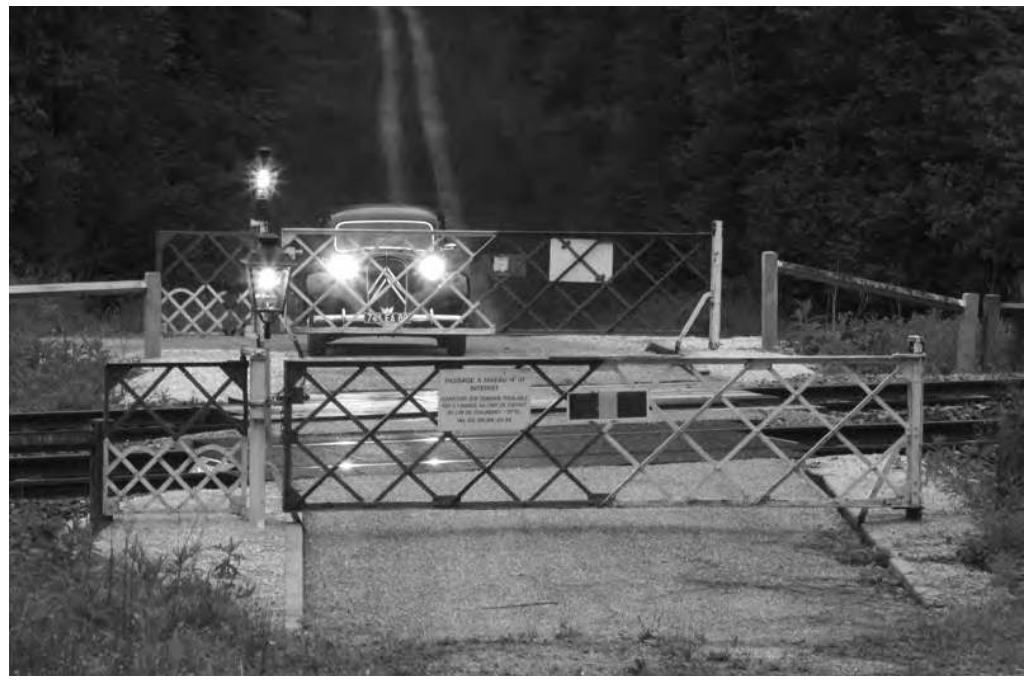

- Figure 8. Reconstitution : passage à niveau allumé au pétrole avec une traction Citroën. ${ }^{\odot}$ et $\mathrm{cl}$. Didier Leroy.

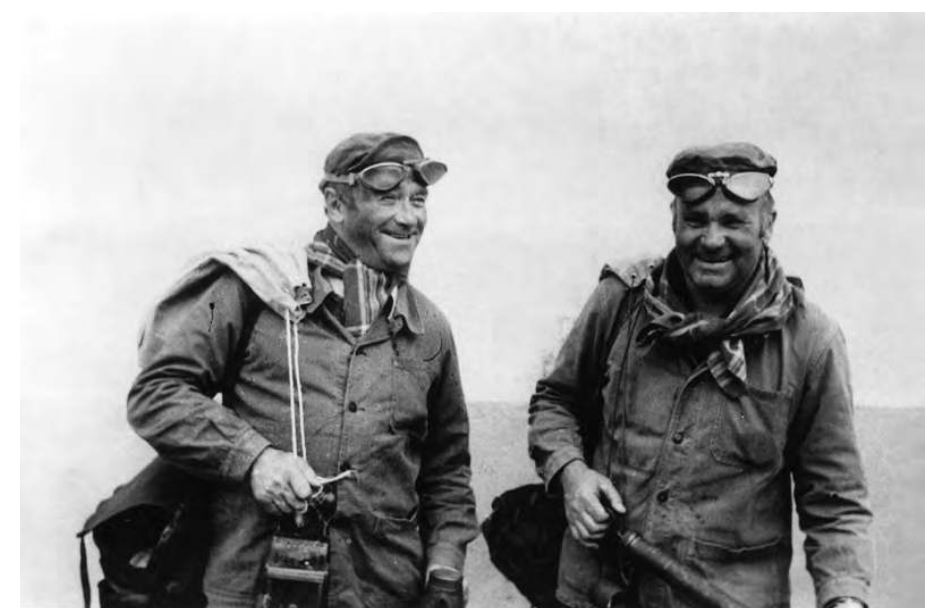

- Figure 9. Le mécanicien et son chauffeur sur la ligne du Train ThurDoller-Alsace, cliché emblématique de l'époque de la vapeur. Cl. d.r., coll. Didier Leroy. 


\section{Annexe}

Inventaire du petit matériel d'une gare type B ( $6^{\mathrm{e}}$ classe/ $/ 5^{\mathrm{e}}$ classe) de la Compagnie de l'Est d'après un récolement du 3 février 1962. Il est à remarquer que l'établissement possède encore une majorité du matériel estampillé « Est ». Les chiffres entre parenthèses correspondent au nombre d'objets. (fig. 10).

Figure 10 •

Livret d'inventaire du petit matériel $\mathrm{d}^{\prime}$ une gare type $\mathrm{B}\left(6^{\mathrm{e}}\right.$ classe $/ 5^{\mathrm{e}}$ classe) de la Compagnie de l'Est, 1962.

${ }^{\circ}$ et cl. Didier Leroy.

\section{Bureau du chef de gare :}

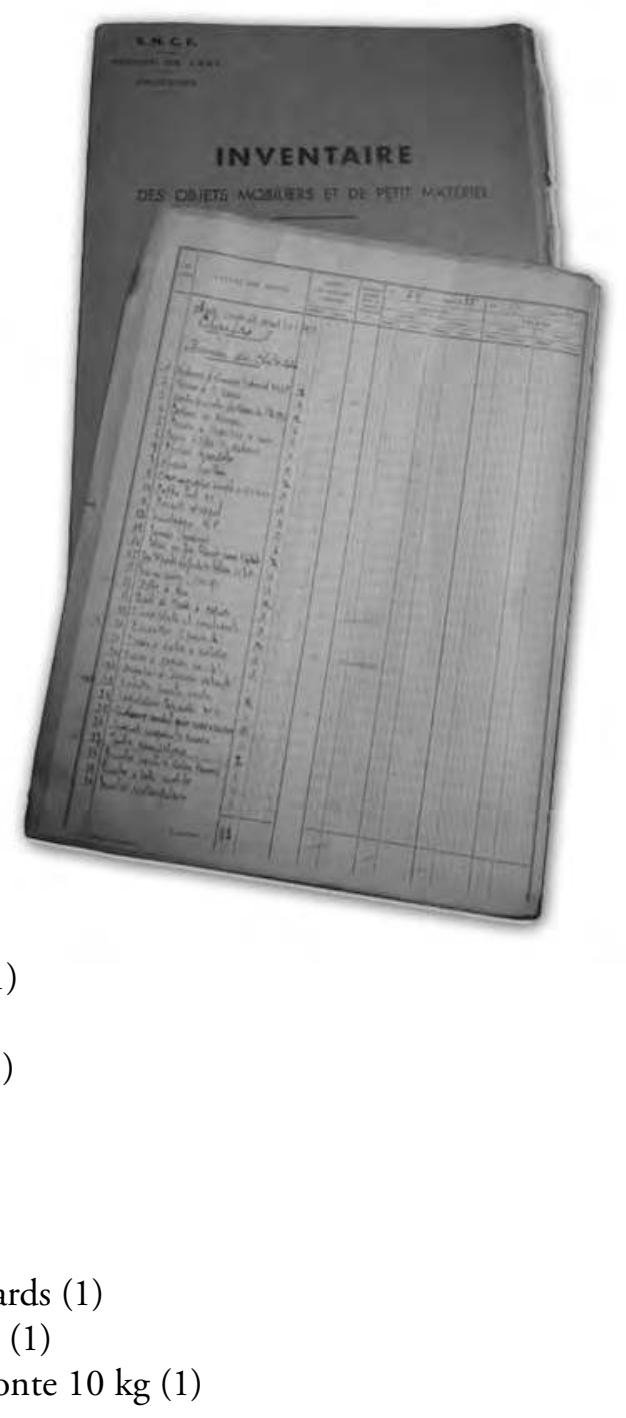


Pince de contrôle gare indice VV (1)

Pied de presse à copier (1)

Presse à copier (1)

Presse à dater à molettes (1)

Régulateur $n^{\circ} 2$ (2)

Rideau coutil gris (1)

Support magasin 5 tiroirs (réserve à billets) (1)

Table hors type (ancienne table du télégraphe) (1)

Timbre cuivre à date mobile (1)

Timbre cuivre enfant gratuit (1)

Timbre cuivre F 717 (1)

Timbre cuivre ovale « Chemin de fer de l'Est - Station de

Timbre cuivre rectangulaire (griffe

Timbre cuivre retour à .......... (1)

Tisonnier en fer (1)

\section{Bureau messageries :}

Balance Roberval de $10 \mathrm{~kg}$ (1)

Bascule romaine de $500 \mathrm{~kg}(1)$

Casier à étiquettes 200 cases en bois (1)

Casier à étiquettes type fer (1)

Chaise paille (dessus bois) (2)

Drapeau rouge grand (2)

Drapeau rouge petit (1)

Pelle à houille (1)

Pelle à main (1)

Pince de contrôle - trou triangulaire - (annulation des billets) (1)

Pince à plomber (1)

Pot à colle en zinc petit type $\mathrm{HO}^{\text {bis }}(1)$

Seau à houille (1)

Série de poids en fonte pour bascule de $500 \mathrm{~kg}$ (1)

Table bureau sans case (1)

Timbre caoutchouc "1.22.21 " (code administratif de l'établissement) (1)

Timbre caoutchouc S.N.C.F. (1)

Timbre cuivre à date mobile et $\mathrm{n}^{\circ}$ train (1)

Timbre cuivre Bagages (1)

Salle d'attente et vestibule :

Armoire à imprimés comportant 5 tablettes (1) 


\section{Quais et voies :}

Arrosoir de quai (1)

Bâche de quai (prélart ou bâche pour couvrir les colis et les bagages) (1)

Banc de la maison «Allez " (1)

Barres d'enrayage [pour immobilisation des wagons équipés de roues à rayons] (4)

Brouette à bagages 1 roue type PO (Paris-Orléans) (1)

Brouette à bagages 2 roues type PLM (Paris-Lyon-Méditerranée) (1)

Brouette à coffre (1)

Cadenas automatiques pour aiguille VU (Voie unique) (6)

Cadenas d'aiguille E (Embranchement) (2)

Cadenas Y (1)

Chaîne pour cadenas de la grue (1)

Char à pont [chariot à 4 roues appelé « Marie-Jeanne »] (1)

Échelle de bâcheur de 4,50 m (1)

Pont de chargement 1er type (1)

Poulain en bois de $3 \mathrm{~m}$ [brancard pour manutention des fûts] (1)

Poulain en bois de 4,50 m (1)

Seau en bois (1)

\section{Halle aux marchandises :}

Anspect freiné de $2 \mathrm{~m}$ [appareil à l'extrémité d'un grand manche en bois permettant la manœuvre à bras des wagons] (1)

Barre pour chargement du charbon (2)

Bascule romaine de $1000 \mathrm{~kg}(1)$

Casier métallique pour étiquettes 10 griffes (1)

Casier pour étiquettes PV 154 cases [PV : petite vitesse] (1)

Cric de $6000 \mathrm{~kg}$ à fut métallique (1)

Diable à farine (1)

Diable à marchandises (1)

Pelle allemande (1)

Pince en fer pied de biche (1)

Pont de chargement 2e type (2)

Pont de chargement 3e type (1)

Pousse-wagon articulé de type cyclo (1)

Raclette triangulaire pour étiquettes (1)

Série de poids de $1000 \mathrm{~kg}$ (1) 


\section{Lampisterie et objets divers :}

Bidon en tôle étamée de $50 \mathrm{~kg}$ type K (1)

Bidons en tôle étamée (pétrole) de $20 \mathrm{~kg}$ (2)

Bidon en tôle étamée (colza) de $5 \mathrm{~kg}$ (2)

Bidon en tôle étamée (colza) de $2 \mathrm{~kg}$ (1)

Boite à carbure de $10 \mathrm{~kg}$ (2)

Burette à long bec (1)

Burette de $2 \mathrm{~kg}$ colza (1)

Burette de $2 \mathrm{~kg}$ pétrole (1)

Ciseau de lampiste (1)

Crible à carbone (1)

Entonnoir à carbure (1)

Entonnoir à pétrole petit (1)

Lampe haute tige au pétrole type $\mathrm{AR}^{\text {bis }}$ [lampe de bureau] (3)

Lanterne extérieure au pétrole type $\mathrm{AF}^{\text {bis }}$ [applique de quai] (2)

Lanterne de signaux d'aiguille au pétrole à flamme jaune type $\mathrm{B}$ [signal de position d'aiguille] (2)

Lanterne à acétylène type YA [lanterne à main] (2)

Marteau (1)

Spatule (1)

Tenaille (1)

\section{Compte trains :}

Clé de fourgon (1)

Édicule d'aisance :

Patère pour WC (3) 
